\title{
Computer vision supported by 3D geometric modelling
}

\author{
Sven Fjeldaas
}

Received: 9 January 2014/Accepted: 18 February 2014/Published online: 13 March 2014

(c) Shanghai University and Springer-Verlag Berlin Heidelberg 2014

\begin{abstract}
This paper suggests four data structures, one based on the other, for a geometric modeller suitable for computer vision. The structures are the key to advance from two-dimensional to three-dimensional automatic picture processing. Three application areas are indicated: one in semisynthetic design of materials, another in measurements of large three-dimensional industrial constructions, and the third one in robotics. There are comments on the software development needed.
\end{abstract}

Keywords Picture processing $\cdot 3 \mathrm{D}$ geometric modelling · Measurements

\section{Introduction}

Picture processing and computer graphics can be traced back to a period shortly after the Second World War when the British needed simulations of radars reporting aircraft activity. It is possible to indicate additional origins as well.

The so-called "mini-computers" of the 1970s came in sizes comparable to a piano and could operate in an ordinary office environment. They had enough computing power for simple computer graphics. Suitable equipment for basic picture processing, as for instance single line CCD cameras and single board computers could be bought off shelf from the early 1980s. Both disciplines, computer graphics and picture processing, are benefitted from

\footnotetext{
S. Fjeldaas $(\bowtie)$

Department of Engineering Design and Materials, Norwegian

University of Science and Technology, Rich. Birkelands vei 2,

7491 Trondheim, Norway

e-mail: Sven.Fjeldaas@ntnu.no
}

available and reasonably priced hardware. Nowadays they are mature and have widespread applications.

However there is a feeling that picture processing may not yet have reached its full potential, especially not in mechanical industry. One may also debate whether computer graphics, now in the shape of geometric modelling, is used as extensively as it could and should be in estimating the properties of materials and in three-dimensional measurements.

This paper proposed the improved ideas on how picture processing and geometric modelling combined could support two entirely different fields of technology: material science and geometric measurements. There appears to be little if any connection between these two fields, but they are both of significant interest to mechanical industry.

\section{Analysis of the distribution of light: "improving" pictures}

Computer programs can "improve" pictures to better serve some specific purpose. The more common improvements are enhancement of highlights and shades and general "sharpening" of pictures. Colours can be added to monochrome pictures, and existing colours can be changed. Films are series of pictures or "frames", and these can be given consistent changes. Shifts and tilts of consecutive frames are adjusted by comparing positions of equivalent details, thus stabilizing the scenes. Footage from the First World War for instance can now be shown with a sharpness, stability, and colour it has never had before [1-3].

A computer program for such services normally attends to all pixels in a frame, one by one, and evaluates neighbour pixels within a suitable distance. Programmers will benefit from notions of patterns like those shown in Fig. 1. 

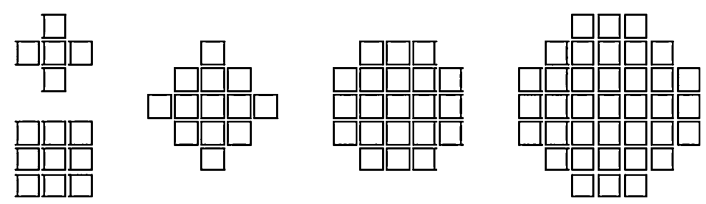

Fig. 1 Patterns used to organize the memory addresses of neighbour pixels

The centre of a pattern is steadily repositioned to match the pixel in focus. The rest of the pattern relates positions of neighbour pixels to their computer memory addresses. All neighbours in the pattern used are inspected. A considerable computing power is therefore needed. Consequently software for computer vision should be designed to avoid repeating these calculations.

\section{2D analysis of shapes in binary pictures}

Living creatures do recognize things and persons on sight. Humans even extract large quantities of information about the real world from two-dimensional pictures and films. However, the processes behind human vision are not well understood. Natural processes can hardly be copied. The alternative is a mathematical approach.

It is common to start with an "improved" picture reduced to a monochrome variant. It is compared to a threshold level. Areas brighter than the threshold are labelled "white". The rest is "black". The result is known as a "binary picture". In most cases black areas are seen as representing solid matter and then to carry the information wanted, but one may of course equally well focus on white areas.

Algorithms can find characteristics of a binary picture [4]: the number of areas and the nesting of areas beside and inside one another. The nesting is described as a hierarchy. Individual areas are commonly characterized by their sizes, parameters of inscribed and circumscribed circles and $X Y$ oriented rectangles, coordinates for the centre of gravity, the second order mechanical moment with respect to the centre, contour length, and the maximum and minimum length vectors from centre to contour. The angle between the two vectors and the second order moments related to these vectors may also be of interest, as are inscribed and circumscribed rectangles with main axes along the maximum vectors.

The process evaluating the characteristics mentioned must necessarily search for and find all pixels defining the contours of the areas. Contour pixel is a needed basis for the characteristics. The contour pixels themselves are summed up as contour lengths. In traditional picture processing individual contour pixels are ignored as soon as

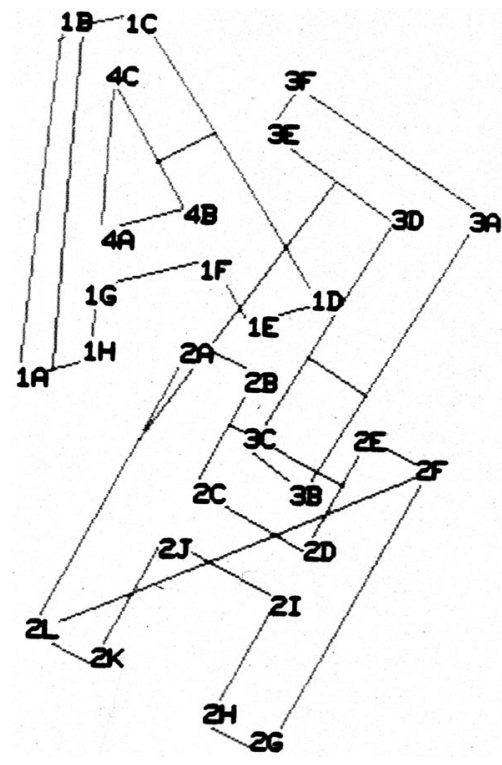

Fig. 2 Positions found and distances evaluated

possible, but the paper will suggest storing their coordinates separately.

An analysis of the set of characteristics may be the desired end result, but the characteristics are often used to recognize pictures and areas within a picture.

Sets of characteristics from all colour components, from several selected threshold levels, and from different "improvements" of the original picture combined will in principle give much information about the scene, but developing a general procedure for extracting meaningful information from more than a single well prepared binary picture is difficult. Nevertheless "3D information from flat pictures" in Sect. 9 is a sketch of a possible methodology.

\section{A first, rudimentary, 2D model}

In mechanical industry distances and angles, as seen in engineering drawings, are considered more relevant than the characteristics mentioned above. The characteristics and the associated recognition is nevertheless valuable support for a computer program searching for coordinates from which it can extract distances and angles. Figure 2 shows a simple example.

Mechanical engineers tend to doubt that the resolution of a camera is sufficient for the accuracy needed for their types of measurements. They may will be right, but the point here is that computer programs will search repeatedly for precisely positioned geometric details in an advanced picture analysis.

All geometric details are on the contours of areas. Finding details is not possible before the relevant contour is 


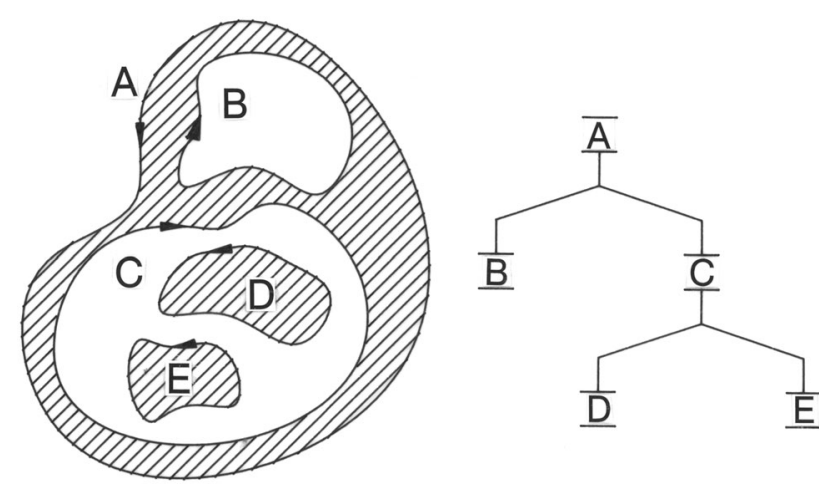

Fig. 3 Hierarchy representing the nesting of contours

determined. Filtering out the pixels defining contours in general calls for an inspection of all pixels and their closest neighbours. This is a time consuming task. It should preferably be done once only.

It is therefore reasonable to store the coordinates of pixels defining contours immediately when found. A container from the $\mathrm{C}++$ library, for instance a "vector", makes a suitable storage [5]. It will provide the coordinates without delay whenever needed. There should be one container per contour.

The interior of an area in a binary picture is uniform and therefore it holds little information. All information for an area can be reproduced and extracted from a detailed description of its outer and inner contours. All remaining picture processing can, if found practical, be based on the stored coordinates.

The set of containers for contour coordinates is a rudimentary geometric model of a binary picture. The hierarchical description of contour nesting can be used to organize the containers. Other characteristics found, areas, centres, second order moments, and so on, are mainly associated with individual contours. Data fields for these are therefore connected to the containers. A software project aiming for 3D picture processing should start with the arrangement indicated above.

Figure 3 shows a simple binary picture with nested areas and a corresponding hierarchy. There are black areas and white ones. An area is identified by its outer contour. Initial characteristics refer to this contour. Characteristics that apply to the entire hierarchy or parts of the hierarchy, as for instance a solid area, are found by combining the initial characteristics from two or more levels of the hierarchy.

A block of data for each item in the hierarchy is useful. In the C programming language a "struct" is a natural choice. In modern $\mathrm{C}++\mathrm{a}$ "class" is more convenient [6]. The variables per contour in the first, rudimentary, 2D geometric model are contour identity, contour type: black or white area, pointer to $\mathrm{C}++$ std::vector of contour coordinates, contour characteristics: length, area, centre of gravity ...

Contour identity and type can be extracted from implicit knowledge, but it is practical to have this information clearly stated. The std::vector pointed at is one of the more commonly used containers from the $\mathrm{C}++$ Standard Library, not the set of coordinates known from vector algebra. The $\mathrm{C}++$ vector can replace a plain array. The advantage added is that the vector can handle dynamic changes in the number of elements. Experienced $\mathrm{C}++$ programmers, familiar with "templates" and "iterators" [7-9], can either store elements inside the vector or alternatively store them individually in the computer's general memory. In the latter case the vector will hold no more than pointers to the elements. The elements used here in the first geometric model are two-dimensional integer coordinates along the contours.

\section{A second, simple, 2D model}

The contour coordinates in their natural form may be somewhat detailed for further use. Coordinates for corners may only be suitable for most purposes. Stretches between corners are then understood as straight line segments. Recognizing corner positions will call for some logic and perhaps even a formal language [10].

Accuracy can be improved by regression analyses of subgroups of contour coordinates followed by calculation of corner positions as intersection points for successive straight lines. In software for a two-dimensional system corner coordinates can be stored in containers equal to or similar to those used for contour coordinates. Original corner pixels are given by integer coordinates, but corner positions calculated to higher accuracy are better saved as floating point numbers.

A second, simple, 2D geometric model should have the collection of variables listed in Sect. 4 extended with a "Pointer to C ++ std::vector of contour corners". The elements of this vector should naturally hold corner coordinates in floating point numbers. Adding the index and the integer coordinates of the closest pixel, as found in the vector of contour coordinates, will speed up the estimation of distances and differences later on.

The process filtering out corner positions opens for an additional type of characteristics describing contour curvatures. It is possible to find convex and concave parts, right and left turns, turn angles, and the distances between these details. Traditional characteristics are single numbers. Contours should be described by sequences of types and magnitudes for characteristics. The sequence length will depend on the complexity of the contour. Single numbers characterizing the complexity will be of interest. 
Contour characteristics should naturally be stored. It is reasonable to store characteristics per straight line segment, later to be called an "edge".

From a formal point of view, a collection of containers holding corner coordinates for contours is a geometric model of a binary picture. It is organized by a hierarchy describing the nesting of contours and has additional data fields of characteristics, but otherwise it may appear simple compared to commonly used geometric models. Some services traditionally handled by specialized data structures, as for instance adding, removing, and substituting elements, are however built into $\mathrm{C}++$ containers. The type of model suggested here is then sufficient for the present purpose and analyses in a two-dimensional world. It is a natural second step for a software project.

\section{A first 3D model: planar polyhedra}

The section will discuss how a picture processing program can establish geometric models of flat binary pictures in data structures suitable for later expansions into solid modelling.

Sufficient data for controlling the expansion are not present in a single binary picture. Some extra information is needed. Reasonable assumptions, as indicated in the next paragraph, will be helpful. Measurements can lead quite far. Measurements of positions by means of photogrammetry will be commented briefly in connection with Fig. 6 . Hints on the third dimension can be extracted from several "improvements" of a "natural" picture to some extent. Some basic procedures are referred to these in Sect. 9.

Suppose that an "improved" aerial photo shows buildings as black areas. It is then relatively simple to "extrude" the contour corners saved in the two-dimensional model to suitable heights, send all coordinates through a multiplication with a transformation matrix, and finally display the scene as if observed from the ground. The two-dimensional model appears as if it is three-dimensional.

Complex models, as for instance of houses with traditional roofs, however cannot be made by extrusions alone, some more advanced tools will be needed. Problems of this kind were partly solved in early computer graphics by introducing three-dimensional "wireframe" drawings, and then wireframes with side panels called "faces" [11-13]. A suitable arrangement of the underlying data, now known as the "Winged Edge Structure", was published by Baumgart in 1972 [14]. A period with many suggestions for generalizations and improvements of this kind of structure followed [15-17]. Competing structures were generally introduced under entirely different names. The period ended with a shift of interest towards Bézier curves and NURBS [18].
The original wireframes are now replaced by complex data structures, often referred to as the "geometric database". There are few structures, if any, left in computer graphics as such. OpenGl for instance manages with two strips of polygons as the most complex items [19]. The databases providing input to computer graphics now belong to the field of geometric modelling.

The two-dimensional geometric model outlined above is easily converted to a three-dimensional "Winged Edge" type of structure, and a modern variant is desirable. A subroutine inspecting the second 2D model and calling the corresponding "Winged Edge" type operations should be developed.

Corner coordinates are given as an extra Z-component and then installed as a "vertex". Installation is done by calling a constructor for a vertex class. Vertices should have extra elements referring to the corresponding contour and perhaps also indices referring to matching positions in the contour- and the corner vector.

The straight line segments from corner to corner had no explicit representation in two dimensions. In three dimensions there is an "edge" element for each of them. Edges are also made by constructors. In a basic implementation the call takes four parameters: head vertex, tail vertex and two faces. In a contour, which is a closed curve, it is reasonable to let the head vertex of one edge become the tail vertex of the next. The inside of a contour becomes two "faces", one on the topside and the other on the underside.

In some applications it is desirable to move contours in a three-dimensional space. This calls for adding transformation matrices to the contour characteristics. If so, one may be best served by a local coordinate system per contour, for instance one rooted in a specific vertex or in the centre of gravity. Coordinate values are changed accordingly. Element values of transformation matrices are set to balance the changes.

\section{Expansions into solid modeling}

The gain obtained by this conversion into a "Winged Edge" type of structure lies in two details. First, all corners, or vertices, can take any number of extra edges. The extra edges can have directions as found useful in the threedimensional space, and the vertices at the far ends of the edges can take new connections. Secondly all edges are connected to two faces, one on either side. In sum these faces make a closed volume. The structure is a general tool for three-dimensional geometric modelling of planar polyhedra, preferably convex ones. Convex planar polyhedra can make acceptable representations of a wide variety of forms.

Concave polyhedra can naturally cover a wider class of objects, but they need a more sophisticated display 
algorithm. Hollow objects can be represented if a hierarchical structure for inside/outside relations is added. Holes through surfaces are normally restricted to be inside single faces, not crossing edges. Holes will call for yet another hierarchical structure, comparable to the one for nested areas in a binary picture. Alternative arrangements have also been used. One may for instance make contours of hole parts of faces' contours by joining these by extra nodes and edges [20]. Such implementations depend on a rule saying that the extra nodes and edges should in most cases be ignored.

There was a tradition for building polyhedron models by means of "Euler operations" [21-23]. These are in principle made for successive subdivisions of a sphere, ensuring that the model at all stages of development is a valid polyhedron. A two-dimensional area is then a sphere made of an upper and a lower half pressed flat. Such "spheres" are allowed to have rather distorted and irregular geometric details. "Euler operations" imposes some constraints on the algorithm building the polyhedra. They also tend to be somewhat demanding with respect to computer resources.

Starting with a minimum of a pure wireframe and then adding vertices, edges, and faces in a random sequence are more convenient in automatic picture processing. The data structure is then ill-defined through longer periods. Some types of "Winged Edge" structures are not designed to operate in this way. Suitable subroutines are not freely available. A picture processing project should be prepared to do most of the source code development.

\section{A second 3D model: sculptured surfaces}

Vertices, edges, and faces may not give sufficiently smooth surfaces in all applications. If so, proper sculptured surfaces are needed. Sculptured surfaces are made by combining "patches". There will be one patch per face. The polyhedral faces are preferably arranged so that each has four vertices. Some faces will then have three.

\subsection{Curves at the edges}

Collections of original contour coordinates selected to match single edges can be sent through a second regression analysis making a best fit of "NURBS", or "non-uniform rational B-splines". The mathematics behind the analysis is commonly referred to as "reverse engineering".

The result of the reverse engineering will be coordinates for points in a "control polygon", represented by floating point numbers. A polygon defines a smooth curve passing through or close to the points and not too far from the edges of the polygon. The finer details of the mathematics are in most cases set so that a curve passes through the first and the last points, but not the others. NURBS keeps their shapes if the control points are moved in space by means of ordinary transformation matrices.

Three-dimensional control points should be kept in containers similar to those used for two-dimensional contour coordinates and corners. The data structure of the "Winged Edge" type should have one container for control points associated with each edge.

Contours are closed curves. It is possible to represent a closed curve by a single uniform rational B-spline. The control polygon will be a closed circuit and the curve will not pass through any control point. It is advised against this design because it will be desirable to subdivide sculptured surfaces into patches corresponding to the faces of the polyhedron. The preferable arrangement is then to have one non-uniform rational B-spline per polyhedron edge, as proposed.

Neighbour patches representing surfaces should preferably be continuous. The coordinates of the endpoint of one control polygon should then be equal to the coordinates of the start of the next. The standard software design meeting this requirement is a data structure with a single set of coordinate values referred to by both polygons. In this case the start- and endpoints should be separated from the rest of the control polygons and stored independently.

In the design suggested here, based on a "Winged Edge" type of structure, control polygons belong to edges. Edges meet at vertices. It is then reasonable to store startand endpoints in the vertices.

Modern CAD systems rarely use data structures of the "Winged Edge" type. Continuity between patches is usually arranged for by manually controlled "stitching" operations.

Coordinate values for corners of a polyhedron and corresponding start- and end points for polygons will in most cases be somewhat different, with the corners at a slightly longer distance from the centre of the polyhedron. It is then reasonable to store two sets of coordinates with each vertex, that is one for a polyhedron corner and one for startand / or endpoints of NURBS.

Smooth transitions between neighbour patches basically depend on corresponding neighbours control points lying in the same plane, close to a common straight line. An algorithm can arrange the control point coordinate values accordingly, but such algorithms will benefit from welldesigned additional data structures. In some CAD systems one will find corresponding manual facilities under the term "fairing".

\subsection{Standard patches}

A standard surface patch has four corners and then naturally four sides [24]. Two opposing sides serve as directrices for a generator spanning the distance between them. 
The two remaining sides give the shape of the generator in it start- and end positions. A polyhedron modeller, for instance one of the "Winged Edge" types, extended with control points for the edges is nearly fit to handle standard patches.

So far the data structure is symmetric. Additional curves guiding the generator's interior control points are needed. These run more or less parallel to the directrices, and thus belong to two of the four sides of the patch only. The design is then asymmetric, which is slightly inconvenient. However, if all curves involved are of the same type and degree and some of the data for the interior guides are moved from the edges into the corresponding polyhedral face of the geometric model, it is possible to make a symmetric arrangement for the entire collection of control points. For an easy start it is recommended to standardize on four control points in both directions, in sum one in each of the four vertices and four connected to the face.

In general control polygons for curves reappear in control nets for patches. Polyhedra are networks. A properly implemented "Winged Edge" type of structure can therefore administer control nets, even irregular and complex ones. Such arrangements will require additional data items separating elements for planar polyhedra and elements for control networks.

\subsection{Triangular patches}

Rectangular NURBS patches can take more general quadrangular shapes, but they do not degrade gracefully into triangles. Triangles are generally needed to make suitable sculptured surfaces.

A special arrangement of three quadrangular patches can nevertheless pass for a larger triangle. Let two neighbour quadrangles have a short common side and long opposing sides. Then fit in a rhomboid with one of the more pointed corners at the end of the common shorter side mentioned, as shown in Fig. 4.

Alternative arrangements for triangular patches and types of less regular patches are known from commercial CAD systems, but a geometric modeller for picture processing can manage without these.

\section{3D information from flat pictures}

Any binary picture here will give contours represented by flat, or 2D, chains of NURBS where the last control point of one curve is also the first of the next one. The interpretations of the contours however depend on the preprocessing, or the "improvement", of the picture.

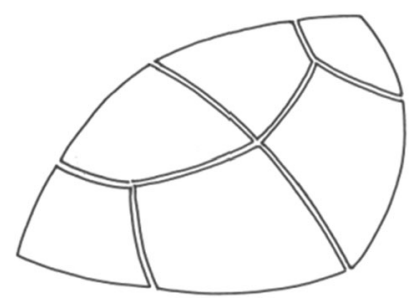

Fig. 4 A pair of triangular patches, each composed of three quadrangles

Suppose that the first "improvement" of a picture enhances the outlines of objects and that a corresponding geometric model has been made. Such initial models will, simply for lack of information, be flat.

The laws of optics are extensively used in computer graphics, not least to render diffuse and mirror reflections. If the second "improvement" of a picture brings forth all highlights and deep shades, it is possible to use the laws of optics "in reverse" to estimate the curvature of surfaces. Polyhedral faces of the initial model are split by introduction of new vertices and edges. $X, Y, Z$ coordinates of vertices are set to reasonable positions. The model's NURBS surfaces can be adjusted accordingly. The result will be far from perfect, but it is a first approach towards a three-dimensional model of the scene rendered by the picture.

The third "improvement" of the picture, focusing on significant changes in gradients not associated with highlights and shades can give further refinements of the curvature.

If the fourth "improvement" assigns equal light values to areas originally having nearly the same gradient, it should be possible to single out surface parts that are close to planar.

It is then reasonable to draw on general knowledge. Manmade objects for instance tend to have several right angles and this will influence estimation of shapes. Research in this area should start with pictures of cubes, pyramids, and other polyhedra and then continue with houses and vehicles. Biological shapes will be significantly more demanding.

Analyses of colour components in the picture can make surface structures appear clearly, and matching geometric models can be built. These additional models are later used to decorate NURBS surfaces by "drawing" on them. Vertices and control points in later variants for "drawing" will hold surface parameter values, traditionally called " $u$ " and " $v$ ". (An extra, the third dimension " $w$ " for the distance to the surface is conceivable). Corresponding $X, Y$, $Z$ coordinates from the NURBS patches are sent to the rendering software, which in turn will reproduce the 
surface structures. Letter shapes will make suitable test objects. A "Winged Edge" type of structure for "drawing" is to be kept separated from those for polyhedra and control networks.

\section{Export to commercial systems}

The proposed data structure, a modern "Winged Edge" type extended with control points for NURBS patches may not fit the ultimate geometric modeller, but that is not the purpose. The data structure is chosen because it can be operated automatically from picture processing algorithms. Making a subroutine interface exporting geometric models from this specialized modeller to commercially available $\mathrm{CAD} / \mathrm{CAM} / \mathrm{CAE}$ systems is a straight forward job. The idea is then to use the CAD/CAM/CAE system for manually controlled enhancements and not least for a variety of analyses, most of them based on finite element methods.

Readers can find a good introduction to the current state of affairs in Zeid [25]. Part II of Ref. [25] is on geometric modelling, and Chapter 9 there, "Solids" comments on data structures now are used by commercial systems.

\section{Application areas}

\subsection{Slices, layer modelling}

In laboratories for materials science grinding and polishing samples is a common procedure. After a finishing procedure, for instance etching, the result is inspected in a microscope. Corresponding pictures can be taken. Grains, fibres, and irregularities showing on the surface are of special interest.

Surfaces of other slices, situated on lower levels, are revealed by repeating the procedure. Marks left by grains, fibres, and irregularities can be traced through several layers.

The picture processing discussed above can build a geometric model of each slice automatically. These models are in principle three-dimensional, but they contain coordinates from flat surfaces only. Combining these models is possible, but there are a few details to handle.

Keeping the original data and data structures for all layers is probably not necessary. Several consecutive layers can be combined into a limited number of NURBS patches.

A contour that appears without a predecessor is probably the top of a detail not seen before. It may be the start of a volume. In surfaces to follow a volume with a simple shape can be expected first to show growing contours, then shrinking contours, and finally nothing. Volumes of complex shapes may however branch off in several directions and they may join other volumes starting higher up.
Establishing the corresponding connectivity can be demanding.

Procedures for picture processing have been applied to horizontal slices of a volume. The connections now sought are in vertical slices. Rules for pixel connectivity should be the same horizontal and vertical. The software analysing horizontal slices can therefore just as well analyse vertical ones.

However, contours are continuous and closed. If one pixel in the circumscribed area is connected to the layer above they all are. An algorithm evaluating vertical connectivity can therefore be made simpler than the one used horizontally.

Coordinating the two directions of analysis and giving a unified presentation of the total result are not quite simple. Among other challenges, the hierarchical structure for nesting of areas in one slice should be connected to an additional structure for connectivity in three-dimensional space.

A hierarchical structure is insufficient in three dimensions. It cannot even represent one of the simpler geometries, a solid ring, also known as a "torus". The structure representing the connectivity should be of the network type. Fortunately, a subset of the "Winged Edge" type, making use of vertices and edges only, can handle the case.

Joining two consecutive contours is quite simple if the contours have the same number of corners. The result will resemble a prism. With a different number of corners some vertices will have more edges connected than others. This calls for a reasonable matching, but which one may not be evident.

Generating connecting edges is a task for the geometric modeller. The algorithm needed can be developed and tested without real pictures. The starting point is a set of models of contours, and these can be coded by hand. Contours of upper- and lower case letters are suitable for initial work. A few simple experiments are illustrated in Fig. 5. The contour of an " $A$ " is extruded and the passage through it is moved outside. An " $H$ " and an " $L$ " are reoriented. The " $\mathrm{H}$ " is given an extra corner and the " $\mathrm{L}$ " is used as a basis for an oddly shaped pyramid.

Complex solids may split, or fork, into smaller branches and smaller parts of a solid can join to form a main body. Polyhedron models need some preparations to handle a split, otherwise the branches will not attach properly to the main body. The main body should have a connection face for the "root" face of each branch. These should match exactly. Connection faces will replace corresponding original faces. They are linked into the polyhedron for the main body by adding edges and filling in auxiliary faces.

A simple procedure joining two parts starts by removing the connection face of the main body and the root face of the branch, leaving two equal contours of vertices and edges. The main body and the branch are then temporarily 


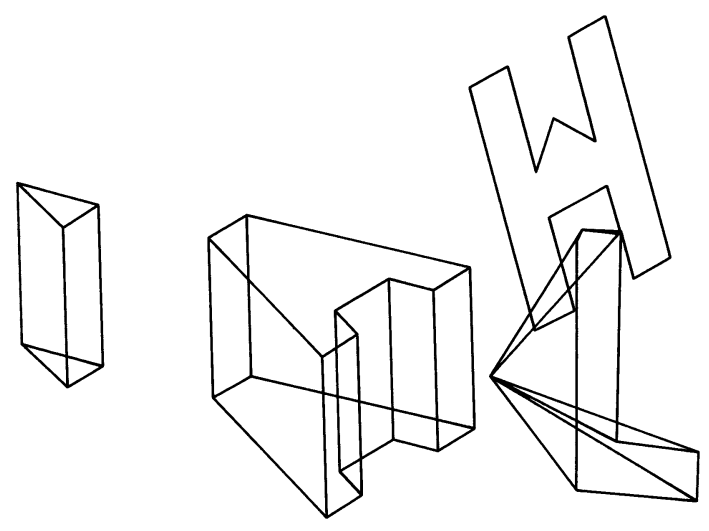

Fig. 5 Experiments with letter-shaped contours

invalid polyhedra which are proceeded by merging matching vertices in the two contours. One set of edges, for instance the one belonging to the branch, is removed in the process.

\subsection{Semisynthetic materials design}

Suppose that a full 3D solid model of a material, showing internal grains, fibres, cracks, and other irregularities, has been transferred to a commercial CAD/CAM/CAE system. The material's elasticity and strength can then be estimated by finite element analyses. Estimation can be calibrated against physical test results.

An engineer may want a material having similar but nevertheless different properties. Properties can be expected to change with adjustments of grains, fibres, and irregularities, and the engineer may have reasonable ideas about changes leading in the right directions. The solid model can be adjusted accordingly and then run through yet another analysis until a satisfactory result appears. The next natural step will be to ask a researcher in material science if a corresponding material can be made.

Inspiration for alternative combinations of material science, picture processing and geometric modelling can be found in Ref. [26].

\subsection{Industrial measurements}

3D vision usually means a stereoscopic view, using two cameras with parallel fields of view and a minor distance between their lenses. In technical applications aiming at measuring distances and angles the cameras may however see one and the same object from entirely different points of view and with an arbitrarily chosen angle between their optical axes. The camera arrangement and the corresponding calculations are in most cases referred to as "photogrammetry". It is commonly used as an advanced form for surveying of

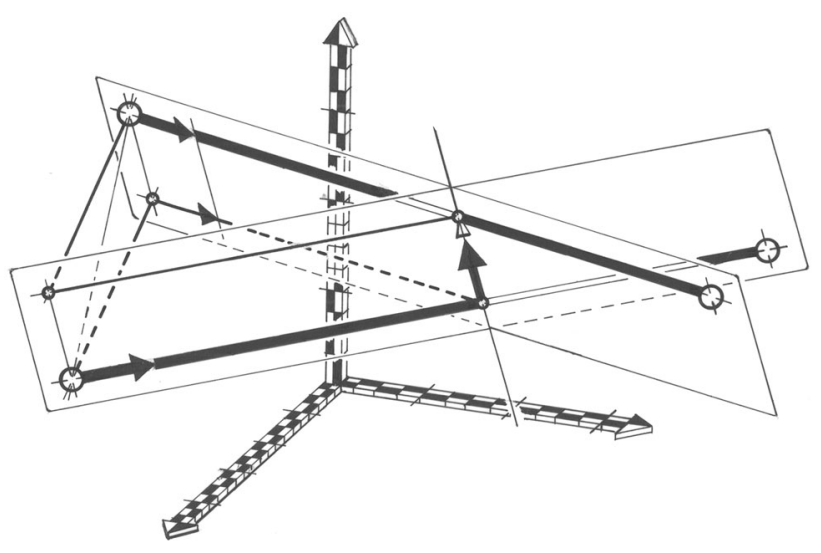

Fig. 6 Two lines of sight and two auxiliary planes

landscapes and also to measure large industrial constructions, as for instance oil rigs made for operations at sea.

The basic problem in "photogrammetry" is to determine the 3D coordinates for the positions of points observed by both cameras. Measurements are calculated from these positions. Calculations fall into one very simple case, point to point distances, and another fairly simple family of cases, corner angles and other characteristics of triangles with corners at given positions, all suspended in a three-dimensional space.

In some carefully prepared cases the camera pictures contain images of clearly marked points only, nothing else. The picture processing needed to find points of interest is then straight forward. If starting with a more common type of image picture processing of various kinds will be needed. Contours given by corner positions, as outlined in this text, may be helpful when searching for details.

Suppose now that pixel coordinates for a point of interest are found for both cameras. Knowing positions and orientations for the cameras two lines of sight can be established through the pixels and the centres of the lenses. These lines of sight should in principle intersect at the position of interest in the three-dimensional space. In practical cases however, the lines will pass one another at some distance. The position of the shortest distance between the lines is given by two points, one on either line. Fortunately the distance and coordinates for the points can be calculated. The procedure is illustrated in Fig. 6. In most cases the midpoint of the shortest distance is the best estimation for the position of the observed point.

Each line of sight is given by its camera position and a unit length directional vector. The shortest distance is perpendicular to both. A vector product of the two directional vectors will give the direction of the perpendicular to them. The length of the perpendicular is the sine value of the angle between them. A scalar product will give the cosine value, if needed. 
Two auxiliary planes defined by the two lines of sight and their common perpendicular are introduced. The planes, the shortest distance, and the camera positions make a triangular prism suitable for further computations.

The shortest distance is found by a scalar product between a unit length common perpendicular, seen as situated at a camera, and a vector from one camera to the other. This distance is also the height of the prism, which leaves two vertices at the camera face of the prism readily at hand. Scale the perpendicular to the length of the distance, add the scaled vector to one camera position and subtract it from the other. Coordinates for one vertex will turn out to be sufficient, for instance the one for the lower triangle. A vector for one triangle edge at the camera face can now be computed and split into a length value and a unit length directional vector.

Scalar and vector products of unit vectors for the sightlines and the triangle's camera side will, with minor post processing, produce cosine and sine values for the two remaining angles of the triangle. A single sine value only is needed. From this value, the sine value at a vertex for the shorter distance, the length of the camera edge and the sine proportion will give the length of either sightline.

A sightline vector is made by scaling the initially given unit vector by the length just found. The vector sum of a camera position, its sightline vector and half of the shortest distance vector gives the estimated position of the point observed. The accuracy of the estimation can be improved by using additional cameras. A comparable approach can be found in Ref. [27].

\subsection{Descriptive geometry in reverse}

Knowing two contours of an object as given by two different camera views and a few common points with their 3D coordinates will in some arrangements be a reasonable starting point for automatic geometric modelling of the original three-dimensional object. The modelling will be a parallel to human interpretation of drawings made in descriptive geometry, in other words descriptive geometry in reverse.

\subsection{Robotics}

The European Commission has a program for the factory of the future [28]. It could cover arrangements allowing robots and humans to work side by side, cooperating. It would of course be nice if industrial robots could see their co-workers and other surroundings and act accordingly. This rather common approach should however be refined somewhat. With a geometric modeller already involved the principles for the refinements is quite simple.
Dimensions of robots are known and bending angles are monitored continuously. The geometric modeller can then keep track of any moving detail of interest. Add instrumentation and Internet connection to human clothing and the workers can be surveyed in the same way [29, 30]. Distances of interest, as for instance the clearing between a human's head and a robot's gripper, can be evaluated in real time. Hazardous situations should then be avoidable.

Static details in "live" pictures are commonly removed by a simple subtraction. Robots and workers move, but they are accounted for by the geometric modeller. The modeller can make a picture of them as seen from any position. Subtracting such carefully calculated pictures from the "live" one will erase men and machines from the scene. The resulting picture shows only the unexpected. It should be thoroughly analysed and used to control further actions. The same procedure will serve several surveillance systems well.

Early work on benefits from combining cameras and robots can be found in Ref. [31]. More recent textbooks in robotics also tend to have a chapter on picture processing [32].

\section{Conclusions}

This paper suggests that a variant of the "Winged Edge" structure should be developed to serve in picture processing, and that the once much used "Euler operations" for such structures should be abandoned. A modern approach can draw heavily on the containers of the $\mathrm{C}++$ standard library.

If contour pixel coordinates are stored along with traditional picture characteristics, a later transition to a threedimensional geometric model of the object seen is possible. The initial part of the process is not too complex. It builds three data structures for the contours. The first is for all contour coordinates. The second is for a best fit of corner coordinates only. Both structures use $\mathrm{C}++$ containers. The third structure is a modern "Winged Edge" type mainly holding the corner coordinates and corresponding edges. This structure can be expanded into a three-dimensional space provided that additional data, not found in a single binary picture, are available.

Methods for expanding the modern "Winged Edge" type of structure into a fourth structure for NURBS patches are indicated. It is recommended to transfer the finished geometric model of the camera scenes to a commercial $\mathrm{CAD} / \mathrm{CAM} / \mathrm{CAE}$ system for further enhancements and analyses.

There are potential applications for functional procedures and software of this kind in automatic surveillance, in control functions for robotics, in measurement 
technology and in semisynthetic materials design. A corresponding development will be demanding but also intellectually rewarding. It is recommended to start by providing a software system for a modern "Winged Edge" kind of structure.

\section{References}

1. The Complete Landmark Television Series (2003) World War in Colour (narrated by Kenneth Branagh)

2. Lindley CA (1991) Practical image processing. In: Arvo J (ed) Graphics gems II. Wiley, New York

3. Niblack W (1986) An introduction to digital image processing. Prentice Hall International, Englewood Cliffs

4. Bieri H, Kohler A (1991) Computing the area, the circumference, and the genus of a binary digital image. In: Arvo J (ed) Graphics gems II. Wiley, New York, p 107

5. Eckel B (2000) Introducing vector. In: Eckel B (ed) Thinking in $\mathrm{C}++$, vol 1, 2nd edn. Pearson Prentice Hall International, Englewood Cliffs, $\mathrm{p} 102$

6. Eckel B (2000) The class. In: Eckel B (ed) Thinking in C++, vol 1, 2nd edn. Pearson Prentice Hall International, Englewood Cliffs, p 270

7. Eckel B (2000) Containers and the template solution. In: Eckel B (ed) Thinking in $\mathrm{C}++$, vol 1, 2nd edn. Pearson Prentice Hall International, Englewood Cliffs, pp 690, 696

8. Eckel B, Allison C (2004) Templates in depth. In: Eckel B, Allison $\mathrm{C}$ (eds) Thinking in $\mathrm{C}++$, vol 2. Pearson Prentice Hall International, Englewood Cliffs, p 227

9. Eckel B, Allison C (2004) Generic containers. In: Eckel B, Allison $\mathrm{C}$ (eds) Thinking in $\mathrm{C}++$, vol 2. Pearson Prentice Hall International, Englewood Cliffs, p 429

10. Denning PJ, Dennis JB, Qualitz JE (1978) Machines, languages, and computation. Prentice Hall College Division, Upper Saddle River

11. McMahon C, Browne J (1993) Wire-frame geometry. In: McMahon C, Browne J (eds) CAD CAM from principles to practice. Addison-Wesley, Boston, p 30

12. Lee K (1999) Wireframe modeling systems. In: Lee K (ed) Principles of CAD/CAM/CAE systems. Addison-Wesley, Boston, $\mathrm{p} 102$
13. Mortenson ME (1985) Wireframe systems" and "graph-based models. In: Mortenson ME (ed) Geometric modelling. Wiley, New York, p 477, 431

14. Baumgart BG (1972) Winged edge polyhedron representation. Stanford University, Stanford. ftp://db.stanford.edu/pub/cstr/ reports/cs/tr/72/320/CS-TR-72-320.pdf

15. McMahon C, Browne J (1993) The surface representation scheme and solid modelling. In: McMahon C, Browne J (eds) CADCAM, from principles to practice, Addison-Wesley, Boston, pp 33, 38

16. Lee K (1999) Winged-edge data structure. In: Lee K (ed), Principles of CAD/CAM/CAE systems. Addison-Wesley, Boston, p 123

17. Lee K (1999) B-Rep data structure and half-edge data structure. In: Lee K (ed) Principles of CAD/CAM/CAE systems. AddisonWesley, Boston, pp 117, 120

18. Piegl L, Tiller W (1995) The NURBS book. Springer, Berlin

19. Woo M, Neider J, Davis T et al (1999) OpenGL programming guide, 3rd edn. Addison-Wesley, Boston, p 45

20. Lee K (1999) One method of treating a face with multiple boundaries. In: Lee K (ed) Principles of CAD/CAM/CAE systems. Addison-Wesley, Boston, p 119

21. Mortenson ME (1985) Euler operations. In: Mortenson ME (ed) Geometric modelling. Wiley, New York, p 420

22. Lee K (1999) Euler operations. In: Lee K (ed) Principles of CAD/ CAM/CAE systems. Addison-Wesley, Boston, p 130

23. Glassner AS (1991) Maintaining winged-edge models. In: Arvo J (ed) Graphics gems II. Wiley, New York, p 191

24. Mortenson ME (1985) Surfaces. In: Mortenson ME (ed) Geometric modelling. Wiley, New York, p 151

25. Zeid I (2005) Mastering CAD/CAM. McGraw Hill, New York, pp 149, 239, 323, 345, 370, 374, 378, 501

26. Lord EA, Mackay AL, Ranganathan S (2006) New geometries for new materials. Cambridge University Press, Cambridge, UK

27. Goldman R (1990) Intersection of two lines in three-space. In: Glassner AS (ed) Graphics gems. Academic Press, Boston, p 304

28. European Commission (2013) The Cordis database. http://cordis. europa.eu

29. Fjeldaas S (2011) Online clothing in NTNU engineering series. In: Dreyer HC (ed) The 13th international modern information technology in the innovation processes conference

30. Fjeldaas S (2008) A framework for exercises in computer science. Comput Appl Eng Educ 16(1):55-61

31. Dodd GG, Rossol L (1979) Computer vision and sensor-based robots. Plenum Publishing, New York, pp 187, 239

32. Spong MW, Vidyasgar SHM (2006) Robot modeling and control. Wiley, New York, pp 377, 407 